\begin{tabular}{|c|r|l|l|l|l|}
\hline Revista Clio América & ISSN: 1909-941X & Vol. 11 & No. 21 & enero - junio de 2017 & 8 - 20 \\
\hline \multicolumn{6}{|c|}{ D0I: http://dx.doi.org/10.21676/23897848.2075 } \\
\hline
\end{tabular}

\title{
Posicionamiento del patrimonio en cooperativas de intermediación financiera frente a las NIIF sobre instrumentos financieros
}

\author{
Positioning of equity in financial intermediation cooperatives compared to ifrs on financial \\ instruments
}

\begin{abstract}
RESUMEN: Esta investigación tiene un diseño no experimental y transeccional; se enfoca en analizar el posicionamiento patrimonial de las cooperativas de intermediación financiera (I.F.) frente a los aportes de sus asociados. Inicia con planteamientos teóricos de normativa contable internacional y local, la relación del Activo total invertido versus el Pasivo, Patrimonio y Aportes. El estudio de caso analizó cifras del balance de situación, las diferencias reales, bajo la incertidumbre de la norma plena. Los resultados arrojan desmejoramiento patrimonial de $14,8 \%$ y endeudamiento general por el mismo orden. En el estudio de caso la situación real, versus la norma plena, y considerando el Capital mínimo irreducible de aportes igual disminuye el Patrimonio en $10.2 \%$ y aumentan los Pasivos Financieros en $10.2 \%$. En conclusión, se considera usar alguna estrategia que solvente la situación de la normativa contable internacional con las normativas nacionales, que converja en beneficio de las cooperativas.
\end{abstract}

Palabras clave: Patrimonio; Cooperativa; Intermediación Financiera; Aportes; Normas Internacionales de Información Financiera NIC / NIIF.

JEL: G, G2, G23

ABSTRACT: This research design is trans-sectional and nonexperimental. The research analyzes the equity position of financial intermediation cooperatives (I.F.) against the contributions of its partners. The paper reviews theoretical approaches of international and local accounting standards, ratio of total invested assets versus liabilities, as well as heritage and contributions. During the case study, the balance sheet figures, and the real differences under the uncertainty of the full standard were evaluated. The results show $14.8 \%$ equity and a similar deterioration of overall indebtedness. Also, in the case study --the real situation--, versus the full standard, and taking into account irreducible minimum capital contributions, Patrimony decreased $10.2 \%$ while Equity and Financial Liabilities increased $10.2 \%$. In conclusion, it is recommended that some strategy be put in place to adjust the situation of international accounting standards to national standards, for the benefit of cooperatives.

Keywords: Equity; Cooperative; Financial Intermediation; Contributions; International Financial Reporting Standards IAS / IFRS

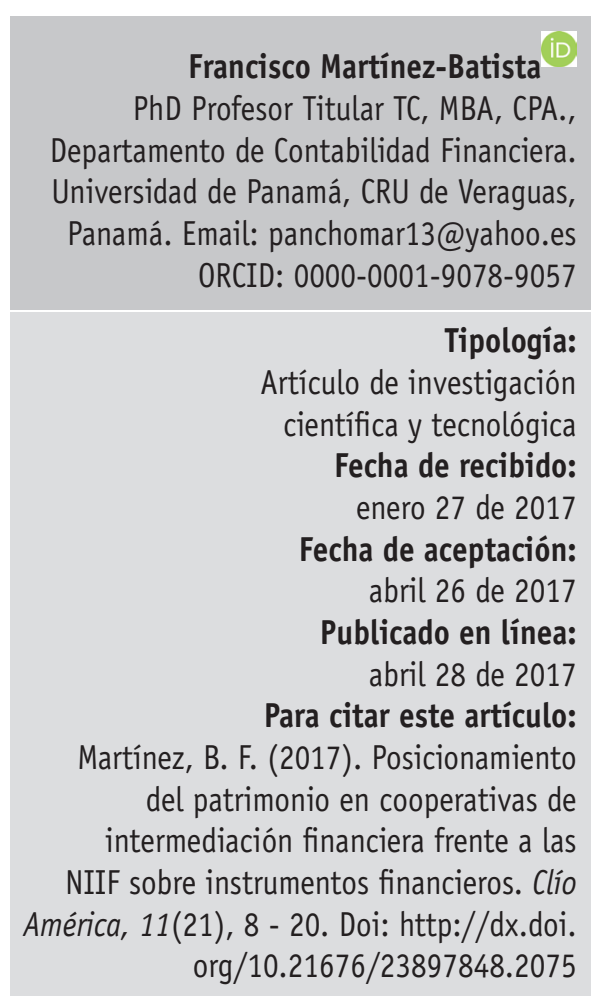




\section{INTRODUCCÍON}

Mucho se ha venido hablando del patrimonio de las sociedades cooperativas. Investigadores contables realizan esfuerzos en homologar el discurso; sin embargo, la filosofía solidaria de las cooperativas crea incertidumbre entre los diferentes planteamientos.

La situación radica, principalmente, en la interpretación (CINIIF 2) ${ }^{1}$ relativa a las Aportaciones de Socios de Entidades Cooperativas e Instrumentos Similares; referenciadas a las NIC 32 y NIC 39². Ésta, pone en perspectiva el dilema de la interpretación: ¿Son las aportaciones de los asociados patrimonio institucional o pasivo financiero? Este estudio tiene como objetivo analizar el posicionamiento del patrimonio de las cooperativas, considerando que el Capital Social (aportaciones de los asociados) de estas promedia el $65 \%$ del total del Patrimonio.

Sin duda que el sector cooperativo, por desarrollarse dentro del contexto social, en donde se aglutinan un número importante de personas, las legislaciones locales (jurisdicciones nacionales) priman en beneficio de ellas creando normas bondadosas.

La legislación panameña salvaguarda, en gran medida, el sostenimiento económico y financiero de las cooperativas, cuando obliga a estas sociedades reservar, al final de los ejercicios sociales anuales, el $10 \%$ para el patrimonio (Ley 17 de 1997). Al igual que el mantenimiento para fines sociales con el $9.5 \%$ de previsión, entre otros aportes patrimoniales propios razonables. Sin embargo, existe una dicotomía entre la legislación nacional y la norma internacional respecto a las aportaciones, cuando establece que "el patrimonio de la cooperativa estará constituido por las aportaciones de los asociados..." (Ley 17 de 1997).

Las cooperativas son sociedades que "están constituidas por grupos de personas con el fin de satisfacer necesidades económicas o sociales que les son comunes" (Comité de Interpretaciones de las Normas Internacionales de Información Financiera

1. En adelante se denominará CINIIF-2. IFRIC, en inglés.

2. La NIC 32 se refiere a los Instrumentos Financieros: Presentación. La NIC

39 se refiere a los Instrumentos Financieros: Reconocimiento y Medición
- CINIIF 2, 2010, p. 2206). La Alianza Cooperativa Internacional para las Américas - ACI américas, define la cooperativa como "una asociación autónoma de personas que se ha unido voluntariamente para hacer frente a sus necesidades y aspiraciones económicas, sociales y culturales comunes por medio de una empresa de propiedad conjunta y democráticamente controlada" (Rendón-Álvarez, Rodríguez-Bolaños \& Riascos-Gómez, 2013, p. 883).

En el contexto cooperativo, grandes organismos estudiosos del tema y de la normativa contable internacional concuerdan en muchos aspectos. Es evidente porque:

Desde la creación de los entes emisores de normas contables, las cooperativas no convergen con el marco teórico de las NIC/NIIF, situación que las ha llevado a usar las normas según las posibilidades y necesidades individuales. Por lo tanto, la normalización contable en el sector cooperativo tropieza por inconveniencias prácticas (Martínez, 2014b, p. 16).

Las diferencias en la aplicación rigurosa de algunas normas, dan resultados desfavorables, en el concierto de la misma normativa, con la realidad empresarial de las cooperativas: valores y principios solidarios (Alianza Cooperativa Internacional para las Américas, ACI-Américas, 2016).

Precisamente, el sector de intermediación financiera se encuentra inmerso en actividades inherentes a las Normas Internacionales de Contabilidad -NIC y las Normas Internacionales de Información Financiera - NIIF3. Se prolija desde el manifiesto de la International Organization of Securities Commissions (IOSCO), en el 2000, cuando destaca y propone "a todos los países miembros de este organismo la utilización de las NIC-NIIF" (Gómez, De La Hoz \& De La Hoz, 2010, p. 152).

Otra acción, en dirección al planteamiento de esta investigación, es fortalecida por la Asociación Española de Contabilidad y Administración quien creó la Comisión de Contabilidad de Cooperativas, misma que ha participado en la creación de la red internacional de investigación de contabilidad para

3. En adelante NIC/ NIIF. En inglés IAS \& IFRS. 
cooperativas y mutuas (The International Research Network on Accounting for Cooperatives and Mutual Entities) (Asociación Española de Contabilidad y Administración - AECA, 2012)

Compartimos que "el principal escollo a salvar se produce por la NIC 32 y NIC 39 sobre instrumentos financieros. La interpretación contenida en la CINIIF 2 no favorece, precisamente, al sector cooperativo..." (Cubedo, 2007, p. 184). También, el discurso, coherente, de la Confederación latinoamericana de cooperativas de ahorro y crédito - COLAC, sobre el riesgo que enfrentan las cooperativas si aplican cabalmente las NIIF que estandaricen la presentación de instrumentos financieros, respecto a una insolvencia. Son situaciones discordantes, de la NIC 32 en las cooperativas, por mencionar algunas.

Se pueden seguir describiendo una serie de estudios, que diferentes organismos y autores han puesto al concurso internacional, pero el foco central en este trabajo de investigación prima sobre el escenario de las cooperativas respecto a su posicionamiento del patrimonio. 0 dicho de otra forma, el efecto del patrimonio tal como lo vienen contabilizando actualmente, su efecto bajo NIC/NIIF (norma plena) y el efecto con la inserción de una alternativa nueva de propuesta modelar MOADANIIF 4 , "se adapten ciertas NIIF a la estructura patrimonial de las cooperativas, dando como resultado la presentación de estados financieros uniformes" (Martínez, 2014a, p. 16).

El Instituto Panameño Cooperativo (IPAC00P), organismo regulador de las cooperativas en Panamá, viene promoviendo que estas empresas apliquen las NIC/NIIF; sin embargo, la realidad nacional (también internacional), es otra; por el hecho de que las aportaciones sociales de los asociados tendrían que ser consideradas Patrimonio si: "a) en caso de retiro del asociado, los aportes pueden ser rechazados por la asamblea, y b) Que no se contemple una remuneración obligatoria al socio o partícipe" (Berrio G., 2015, p. 29). En ambas situaciones, legal y estatutaria, no presentan estas figuras.

4. MOADANIIF es una propuesta modelar, para cooperativas, que pudiera ser considerada por los emisores de normas contables para equilibrar las aportaciones de asociados, la aplicación plena de la norma internacional, y la propuesta basada en Ratios.
Existen dilemas sobre el posicionamiento patrimonial en las cooperativas por los instrumentos financieros, debido a los preceptos que emiten las normas. Al contrastar las NIC/NIIF, en cuanto al mandato y tratamiento contable de los instrumentos financieros (e.g. NIC 32, NIC 39, NIIF 7), como también la interpretación CINIIF 2, con las leyes regulatorias nacionales de las cooperativas, se puede visualizar que los textos distan fuertemente.

Nos permite, entonces, formular la pregunta: ¿Cómo se posiciona el patrimonio de las cooperativas de intermediación financiera frente a las NIIF sobre instrumentos financieros?

Escriba aquí la explicación de los conceptos, enfoques y autores que orientan la investigación.

Para las sociedades cooperativas, converger a las NIC/NIIF se convierte en un tema cuestionado, a nivel mundial, debido a las características propias que presentan estas organizaciones en su estructura patrimonial.

La presente investigación centra su estudio en el análisis de variables que posicionan el valor patrimonial en las cooperativas de intermediación financiera. Analizar variables como Capital Social (Aportaciones), Pasivo Financiero, Instrumentos Financieros, entre otras, determinan las distancias entre la aplicación, o no, de la normativa contable, con la norma legal estatal y la estatutaria institucional de las cooperativas.

La International Accounting Estándars Board (IASB), como organismo emisor de normas contables, en algunos casos, generaliza los conceptos sin mayores consideraciones del tipo empresarial, como es el caso de las cooperativas.

Temas no agotados son sobre si el aporte mínimo e intereses "puede clasificarse como neto" (Polo G., 2007, p. 88; Polo G., 2006, p. 120), 0 si se están aplicando, según el párrafo 8 de la CINIIF 2 , dependiendo de la reglamentación legal local e institucional "pueden imponer diferentes tipos de prohibiciones para el rescate de las aportaciones de los socios" (Comité de Interpretaciones de las 
Normas Internacionales de Información Financiera - CINIIF2, 2010, p. 2207).

Si bien, en la actualidad "el capital social de las sociedades cooperativas están constituidos por las aportaciones, efectuadas para ese fin, tanto de carácter dinerario como no dinerario" (Martin, Lejarriaga \& Iturrioz, 2007, p. 62), su conversión a las NIC/NIIF plenas, podrían presentar impactos negativos en los Estados Financieros; porque "hay contradicciones en la conceptualización de los fondos propios de las cooperativas. Hecho que no ocurre en las sociedades de capitales" (Cubedo, 2007, p. 171).

Para acercamiento de la normativa contable con las legislaciones locales, sobre el tema cooperativo, se trabaja. Es necesario recordar que las NIC/NIIF "son un conjunto de normas de alta calidad que establecen un lenguaje global y común que facilita el reconocimiento, medición y revelación de la información financiera" (Gómez, De La Hoz \& De La Hoz, 2010, p. 150), independientemente donde esté localizado el país.

El campo de acción de las NIC/NIIF incluye, predominantemente, "todo tipo de organización (sociedades de capitales, sociedades solidarias, entre otras); sin embargo, el uso de esta estandarización contable depende de las leyes en diferentes jurisdicciones nacionales, el tipo de organización (nacional 0 supranacional), la actividad empresarial, por mencionar algunas" (Martínez, 2014a, p. 43).

El concepto de las NIIF trata de minimizar el efecto adverso de credibilidad que tiene la normativa contable sobre temas puntuales del usuario de la información, las diferencias contables internacionales, el precio de adquisición, y la contabilidad creativa; es decir, pretende eliminar las múltiples alternativas para contabilizar operaciones que varían radicalmente los resultados de empresas en diferentes países (Amat y Perramon, 2005, p 12).

En consecuencia, la NIC (Normas internacionales de contabilidad) y las SIC (Comité de Interpretaciones de las Normas), son las normas e interpretaciones creadas por los predecesores de la IASB y el Comité de Interpretaciones de las NIIF. Estos entes técnicos fueron adoptados por el IASB y el Consejo IFRS 5 Interpretations cuando se hizo cargo en 2001, y por lo tanto, forman parte del cuerpo de los requisitos de las NIIF (www.iasb.org) (Martínez, 2014a).

Con la adopción de las NIIF en la Unión Europea, las normas que tienen efecto negativo en las sociedades cooperativas, de alguna forma, están siendo estudiadas para adecuarlas a la realidad. Aunque se haya dotado a las cooperativas de normativa específica (e.g. CINIIF 2 o IFRIC 2), flexibilizando algunas aplicaciones por considerárseles no significativas, debido a que la mayoría son pequeñas y medianas empresas.

Respecto al capital social del sector empresarial solidario, entra en juego el uso de instrumentos financieros. De forma abstracta, según la NIC 32 (IAS 32$, p. 1$)^{6}$, "un instrumento financiero es cualquier contrato que dé lugar a un activo financiero en una entidad y a un pasivo financiero o instrumento de patrimonio en otra entidad".

Se entiende como activo financiero:

...el efectivo, instrumento de patrimonio de otra entidad, un derecho contractual (...), un contrato que será o podrá ser liquidado con instrumentos de patrimonio propio de la entidad (...); como pasivo financiero se entiende a una obligación contractual (...), un contrato que será, o podrá ser liquidado, utilizando instrumentos de patrimonio propio de la entidad (...) (IAS 32, 2012, p. 2).

En este contexto (IAS 39, 2012, p. 1), para su aplicación se debe considerar "el deterioro del valor e incobrabilidad de los activos financieros medidos a costo amortizado".

La NIC 32 indica que su objetivo es:

...establecer principios para presentar los instrumentos financieros como pasivos o patrimonio y para compensar activos y pasivos financieros. Ello aplica a la clasificación de los instrumentos finan-

5. IFRS (International Financial Reporting Standard), son las siglas en inglés de las NIIF

6. IAS (International Accounting Standars) son las siglas en inglés de las NIC 
Posicionamiento del patrimonio en cooperativas de intermediación financiera frente a las NIIF sobre instrumentos financieros

cieros, desde la perspectiva del emisor, en activos financieros, pasivos financieros e instrumentos de patrimonio; en la clasificación de los intereses, dividendos y pérdidas y ganancias relacionadas con ellos; y en las circunstancias que obligan a la compensación de activos financieros y pasivos financieros. (IAS 32, 2012, p.1)

Por su lado, "los principios contenidos en esta NIIF 7 (Instrumentos Financieros: información a revelar) complementan a los de reconocimiento, medición y presentación de los activos financieros y los pasivos financieros de la NIC 32 Instrumentos Financieros: Presentación, y de la NIC 39 Instrumentos Financieros: Reconocimiento y Medición" (NIIF 7, 2015, p 657).

Esta norma (NIIF 7) tiene efectos directos con sociedades solidarias ya que es aplicable a todo tipo de empresas, tanto las que tienen pocos instrumentos financieros (e.g. las que solo tienen cuentas por cobrar y acreedores), como las que tienen muchos instrumentos financieros (e.g. las instituciones financieras que casi todos sus activos y pasivos son instrumentos financieros); esto es, "la NIIF se aplica a todas las entidades" (IFRS 7, 2012, p. 655).

La CINIIF 2, relativa a las aportaciones de socios de entidades cooperativas e instrumentos similares, plantea que:

...se aplicará a los instrumentos financieros que están dentro del alcance de la NIC 32, entre los que se incluyen los instrumentos financieros emitidos a favor de los socios de entidades cooperativas, que constituyen participaciones en la propiedad de dichas entidades. Esta Interpretación no será de aplicación a los instrumentos financieros. (Comité de Interpretaciones de las Normas Internacionales de Información Financiera- CINIIF 2, 2010, p. 2206)

La CINIIF 2 indica que:

...Muchos instrumentos financieros, incluidas las aportaciones de los socios, tienen características de patrimonio neto, como el derecho de voto y el de participación en el reparto de dividendos. Algunos instrumentos financieros otorgan al tenedor el derecho a solicitar su rescate en efectivo o mediante la entrega de otro instrumento financiero, pudiendo incluir, o estar sujeto este rescate a determinadas limitaciones. (Comité de Interpretaciones de las Normas Internacionales de Información FinancieraCINIIF 2, 2010, p. 2207)

Existe un criterio crítico, entre otros relevantes también, en la CINIIF 2, respecto a las aportaciones de los asociados, cuando plantea que:

...las aportaciones de los socios por encima del nivel, a partir del cual se aplique la prohibición de rescate, serán pasivos [...] y el importe del capital desembolsado, sujeto a la prohibición de rescate, puede variar en el tiempo. Dichas variaciones darán lugar a una transferencia entre pasivo financiero $y$ patrimonio neto. (Comité de Interpretaciones de las Normas Internacionales de Información FinancieraCINIIF 2, 2010, p 2207)

También, que:

...Los tenedores de instrumentos de patrimonio se reconocerán directamente en el patrimonio neto, por un importe neto de cualquier beneficio fiscal relacionado. Los intereses, dividendos y otros rendimientos relativos a los instrumentos financieros clasificados como pasivos financieros, serán gastos, con independencia de que dichos importes pagados se califiquen legalmente como dividendos o intereses, o bien reciban otras denominaciones. (Comité de Interpretaciones de las Normas Internacionales de Información Financiera- CINIIF 2, 2010, p. 2208)

Como existen cooperativas categorizadas como PYMES, la NIIF para PYMES, tienen iguales condiciones para tipificar la estructura del patrimonio cuando sostiene que las aportaciones de socios e instrumentos similares, son patrimonio si la entidad tiene un derecho incondicional para rechazar el rescate de las aportaciones, y si el rescate está incondicionalmente prohibido por la ley nacional, por reglamentos o por los estatutos de la entidad (Consejo de Normas Internacionales de Contabilidad - IASB, 2016).

\section{METODOLOGÍA}

En la práctica, el patrimonio de las cooperativas, por su característica institucional: 
...está formado por las aportaciones realizadas por uno 0 más sujetos para constituir una sociedad (capital social), semejante al caso del capital en entidades empresariales no societarias. Confiere los derechos sociales (aunque no los estructura en el caso de las sociedades cooperativas). (Gómez A. \& Miranda G., 2006, p. 15)

También, una cooperativa:

... tiene personalidad propia y diferente de los sujetos que la integran, luego jurídicamente los recursos pertenecen a la sociedad. Las características de la "propiedad" del socio son peculiares respecto a otras formas societarias: Asimismo el socio no tiene derecho a "llevarse" gran parte de las reservas (las reservas legales), que se quedan en la sociedad y que no pueden repartirse. (Gómez A. \& Miranda G., 2006, p. 15)

Las Normas Internacionales de Contabilidad, sobre la tipificación de las cooperativas, sostiene que "independientemente de la calificación financiera de las Normas Internacionales de Información Financiera las aportaciones societariamente siguen siendo capital social en sentido jurídico, lo que conlleva una diferente caracterización desde el punto de vista jurídico y contable" (Gómez A. \& Miranda G., 2006, p. 16).

En esa línea, la exigibilidad de un recurso es pasivo financiero cuando existe la obligatoriedad de devolución por la empresa. Cuando no exista exigibilidad ordinaria de rescate, sería un instrumento de capital.

Para determinar el contraste, se sometió a un estudio de caso. Entendiéndose que el estudio de caso puede considerarse como una metodología o como un método: Metodología, porque su enfoque general estudia un problema de investigación; y Método, porque aplica una técnica concreta de recolección de datos. Ambos enfoques se ajustan al proceso metodológico usado aquí, debido a que los datos proyectan descripciones sobre los cambios de las variables explicadas.

La investigación es de tipo exploratoria, descriptiva y explicativa; en el sentido que describe y explica, con mayor profundidad, el posicionamiento patrimonial con respecto al uso, 0 no, de la normativa contable internacional (NIC/NIIF); es decir, estudia la asociación entre estas variables. Tiene un enfoque cuantitativo, por su carácter numérico; entendiéndose que "se basa en el positivismo, según el cual existe una verdad objetiva en torno a las organizaciones que se revela a través del método científico" (Hernández S., Fernández C. \& Baptista L., 2007, p 5).

Su diseño es no experimental y de prevalencia. El mismo, además del estudio teórico, analiza los datos empíricos procesados de los Estados Financieros de las cooperativas seleccionadas que validan los resultados obtenidos.

El procesamiento de los datos fueron realizados de la siguiente manera: 1) Se seleccionaron cinco cooperativas de I.F. más grandes del país. Entre las más representativas, la Cooperativa de Ahorro y Crédito El Educador (COOPEDUC), la Cooperativa de S.M. Los Profesionales, la Cooperativa de S.M. el Educador Veragüense (COOPEVE), la Cooperativa de Ahorro y Crédito San Antonio (CACSA) y la Cooperativa el Educador Santeño (COESAN). 2) Se capturaron los datos originales de sus Estados Financieros auditados del 2015, sobre el Pasivo, Patrimonio, Aportaciones, entre otros datos de las cooperativas participantes del estudio. 3) Se analizaron las variables reales, bajo NIC/NIIF y basada con la propuesta de modelo de adaptación NIIF al patrimonio de las cooperativas. Y 4) Se presentaron los resultados cuya discusión determina los efectos del posicionamiento del patrimonio según la estructura actual, bajo las NIC/NIIF y bajo el criterio de la propuesta modelar de adaptación patrimonial de las NIIF, indicada antes.

\section{RESULTADOS Y DISCUSIÓN}

\section{Posicionamiento actual de las Aportaciones en la estructura del Patrimonio cooperativo}

En términos totales (sector cooperativo nacional), la información financiera real de las cooperativas posiciona B/. 301,628,416 en Aportaciones de B/. 505,423,380 en Patrimonio (Instituto Panameño Autónomo Cooperativo - IPACO0P, 2016), representando el $59.7 \%$ de Aportaciones contabilizado en la estructura del Capital, en el primer semestre 2016, Tabla 1. 


\section{Tabla 1.}

Información financiera (Activo, Pasivo, Patrimonio y Aportaciones) total del sector cooperativo en Panamá. I Trimestre, 2016 (En Balboas)

\begin{tabular}{|c|c|c|c|c|}
\hline \multicolumn{5}{|c|}{ INSTITUTO PANAMEÑO AUTÓNOMO COOPERATIVO } \\
\hline IrUKI & SEGUN TIPC & PERATIVA. EN & ES. I TRIMESTF & 16. EN BALBOA: \\
\hline ipo de Cooperativa & Activos & Pasivos & Patrimonio & Aportaciones \\
\hline Total & 2.037.415.188 & 1.531.991.808 & 505.423 .380 & 301.628 .416 \\
\hline
\end{tabular}

Fuente: Elaboración propia, basada en el IPACOOP

Estos datos presentan la situación real separando el efecto de las Aportaciones en el estudio (7\%). Sin embargo, la situación financiera real, en donde las Aportaciones se integran al Patrimonio, representa el $50 \%$ del Activo, $38 \%$ del Pasivo y $12 \%$ del Patrimonio. Estas cifras corresponden a todo el sector cooperativo del país.
Como el estudio aplica el método de Estudio de Caso de cinco (5) de las cooperativas de I.F. representativas del país, veamos los resultados de sus Estados Financieros del 2015. Primero se presenta la situación real de la estructura patrimonial y los Pasivos Financieros de la muestra (Tabla 2).

Tabla 2.

Situación financiera de cooperativas de I.F. de Panamá -Situación Real -Actual (En Balboas)

\begin{tabular}{|l|r|r|c|}
\hline \multicolumn{1}{|c|}{ NOMBRE DE LA COOPERATIVA } & ACTIVOS & PASIVOS & PATRIMONIO \\
\hline Caso 1 & 341.803 .991 & 320.910 .463 & 20.893 .528 \\
\hline Caso 2 & 339.257 .601 & 262.827 .007 & 76.430 .594 \\
\hline Caso 3 & 202.051 .923 & 144.399 .115 & 57.652 .808 \\
\hline Caso 4* & 141.494 .501 & 119.052 .069 & 22.442 .432 \\
\hline Caso 5* & 60.201 .282 & 51.768 .756 & 8.432 .526 \\
\hline Totales & & 898.957 .410 & 185.851 .888 \\
\hline Porcentaje/Participación (Base = Activo) & 1.084 .809 .298 & $82,87 \%$ & $17,13 \%$ \\
\hline *Son datos del 2012 & & & \\
\hline * *Son datos del 2013 & & & \\
\hline
\end{tabular}

Fuente: Elaboración propia 
En la realidad, las cooperativas sujetas a estudio (5 entre las más representativas en el país), resultan que el Patrimonio representa el $17.13 \%$ del Activo y el Pasivo, $82.87 \%$. La estructura del Patrimonio incluye todas las Aportaciones, Reservas y Excedentes Acumulados. El mismo (con B/.185,851,888) tiene la siguiente distribución: (Tabla 3 ).

\section{Tabla 3.}

Porcentajes de distribución según la estructura real del Patrimonio cooperativo (En Balboas)

\begin{tabular}{|l|c|c|c|c|}
\hline & & PASIVOS & PATRIMONIO & APORTACIONES \\
\cline { 3 - 5 } COOPERATIVA & PATRIMONIO TOTAL & APORTACIONES & RESERVAS & $\begin{array}{c}\text { EXCEDENTES NO } \\
\text { DISTRIBUIDOS }\end{array}$ \\
\hline Caso 1 & 20.893 .528 & 9.632 .530 & 11.051 .870 & 209.584 \\
\hline Caso 2 & 76.430 .594 & 33.785 .530 & $40.763,276$ & 1.881 .788 \\
\hline Caso 3 & 57.652 .808 & 40.679 .623 & 15.587 .163 & 1.386 .022 \\
\hline Caso 4* & 22.442 .432 & 20.369 .278 & 1.411 .095 & 662.059 \\
\hline Caso 5** & 8.432 .526 & 6.602 .985 & 1.666 .743 & 162.798 \\
\hline * 2012 & $185,851,888$ & 111.069 .490 & 70.480 .147 & 4.302 .251 \\
$* * 2013$ & & $59,76 \%$ & $37,92 \%$ & $2,31 \%$ \\
\hline
\end{tabular}

Fuente: Elaboración propia

Es evidente la fortaleza de las Aportaciones (59.76 $\%$ ) en el Patrimonio de los cinco casos estudiados. El resto de la estructura patrimonial (37.92 \% y 2.31 $\%$ ), completan las Reservas totales y los Excedentes no distribuidos, respectivamente.

\section{Posicionamiento de las Aportaciones bajo NIC/NIIF}

Siguiendo la secuencia del apartado anterior, la investigación simula el escenario de aplicación de la normativa NIC/NIIF (e.g. NIC 32 y CINIIF 2), donde, en primera instancia, transfiere las Aportaciones capitalizadas al Pasivo financiero. Bajo esta figura tenemos: (Tabla 4).

Es claro el efecto financiero negativo empresarial, de reconocerse los aportes de los asociados, que están siendo capitalizados, al Pasivo Financiero. Esto es que los mismos estén en disposición de reembolso permanente. En el escenario, que todo el sector cooperativo del país estuvieran bajo NIC/NIIF, disminuirían su capacidad patrimonial de $24.8 \%$ a $10.0 \%$ (14.8 \%), en contraste, se elevarían las deudas institucionales (Pasivo Financiero) de $75.2 \%$ a $90.0 \%$ (14.8\%). 
El posicionamiento patrimonial, en este estudio de caso, aplicando el escenario de las NIC/NIIF e interpretación CINNIF, se enfoca en estimar el Capital Mínimo irreductible concertado en los estatutos de las societarias. Según el número de asociados por una aportación, estima aquí no reembolsable B/. 773,250 del total aportado (B/.111,069,490), representando menos del $1 \%(0.70 \%)$ (Tabla 5$)$.

Tabla 4.

Reconocimiento de los Aportes capitalizados al Pasivo Financiero de todo el sector Cooperativo (En Balboas)

\begin{tabular}{|l|c|c|c|}
\hline \multicolumn{1}{|c|}{ TIPO DE COOPERATIVA } & ACTIVOS & PASIVOS & PATRIMONIO \\
\hline Total general & 2.037 .415 .188 & 1.531 .991 .808 & 505.423 .380 \\
\hline Bajo NIC / NIIF & 2.037 .415 .188 & 1.833 .620 .224 & 203.794 .964 \\
\hline \% Total general & & $75,2 \%$ & $24,8 \%$ \\
\hline \% Bajo NIC / NIIF & & $90,0 \%$ & $10,0 \%$ \\
\hline
\end{tabular}

Fuente: Elaboración propia

Tabla 5.

Estructura del Patrimonio cooperativo considerando el Capital Aportado minimo irreducible (En Balboas)

\begin{tabular}{l|c|c|c|c|c|c|}
\hline \multirow{2}{*}{$\begin{array}{l}\text { CASOS } \\
\text { ESTUDIADOS }\end{array}$} & APORTACIONES & & & \multicolumn{2}{|c|}{ PATRIMONIO BAJO NIIF } & \\
\cline { 3 - 8 } & $\begin{array}{l}\text { CAPITAL } \\
\text { MÍNIMO }\end{array}$ & $\begin{array}{c}\% \text { CAP. } \\
\text { MÍNIMO }\end{array}$ & APORTACIONES & $\begin{array}{l}\text { RESERVAS } \\
\text { Y EXCED. }\end{array}$ & $\begin{array}{l}\text { EXCEDENTES NO } \\
\text { DISTRIBUIDOS }\end{array}$ \\
\hline $\begin{array}{l}\text { Totales de } \\
\text { Casos }\end{array}$ & 111.069 .490 & 773.250 & $0,70 \%$ & 773.250 & 74.782 .398 & 1.009 .253 .650 \\
\hline
\end{tabular}

Fuente: Elaboración propia

El comportamiento, según el escenario anterior, posiciona el Patrimonio de las cooperativas de I.F., en los casos estudiados, con un $6.9 \%$ de Patrimonio y con $93.11 \%$ de Pasivo Financiero. Significa que, con el escenario del mandato de las NIC/NIIF y CINIIF-2, la situación Patrimonial de las cooperativas estudiadas se desmejora en $10.24 \%$, mientras que se endeuda la empresa en la misma proporción (10.24\%) (Tabla 6). 
Tabla 6.

Posición del Patrimonio en cooperativas de I.F. de la Situación Real vs la Situación Bajo NIC/ NIIF) (En Balboas)

\begin{tabular}{|l|c|c|c|c|c|c|}
\hline & \multicolumn{2}{|c|}{ SITUACIÓN REAL AL 31 DIC. 2015* } & \multicolumn{2}{c|}{ SITUACIÓN BAJ0 MANDATO NIC7NIIF } \\
\hline $\begin{array}{l}\text { Nombre de la } \\
\text { cooperativo }\end{array}$ & Activo & Pasivo & $\begin{array}{c}\text { Patrimonio } \\
\text { Total }\end{array}$ & Activo & Pasivo & $\begin{array}{c}\text { Patrimonio } \\
\text { Total }\end{array}$ \\
\hline $\begin{array}{l}\text { Totales de } \\
\text { Casos }\end{array}$ & 1.084 .809 .298 & 898.957 .410 & 185.851 .888 & 1.084 .809 .298 & 1.009 .253 .650 & 75.555 .648 \\
\hline $\begin{array}{l}\% \\
\text { Participación } \\
\text { Base: Activo }\end{array}$ & $82,87 \%$ & $17,13 \%$ & & $93,11 \%$ & $6,89 \%$ \\
\hline $\begin{array}{l}\text { *Caso 4: } \\
\text { Datos 2012 }\end{array}$ & $100 \%$ & & & $100 \%$ & \\
\hline
\end{tabular}

Fuente: Elaboración propia

Posicionamiento del Patrimonio respecto a la propuesta de modelo de adaptación de las NIFF (MOADANIIF) al Patrimonio cooperativo

Para plantear un escenario basado en la propuesta de modelo de adaptación de las NIIF al Patrimonio de las cooperativas (Martínez, 2014a), hay que explicar algunos conceptos de esta propuesta.

El modelo está formulado mediante Ratios ${ }^{7}$, de la siguiente forma: a) De Liquidez (Solvencia a corto plazo): b) De Endeudamiento (Solvencia a largo plazo); y c) De Rendimiento Financiero (ROE: Return On Equity). De estos factores, se desprenden seis variables independientes (categóricas), así:

Factor:

Liquidez (Corto Plazo)

Endeudamiento (Solvencia a Largo Plazo)

Rendimiento financiero (ROE)

Variables:

Activo Circulante / Pasivo Circulante

Pasivo Total / Activo Total

Beneficio Neto del periodo /Capital Contable

(Patrimonio Neto)
Los Estados Financieros deben cumplir con su Marco Conceptual ${ }^{8}$ de quienes va a depender la confianza que se tenga para cuantificar los RATIOS; aunado al cumplimiento de los Principios de Contabilidad Generalmente Aceptados (PCGA) por parte de las entidades.

Los modelos basados en Ratios (e.g. el de Baever), expresan los tres Ratios propuestos aquí. Todos ligados a modelos predictivos.

El procedimiento, relativamente, asegura que los ratios caigan dentro de los rangos considerados óptimos (neutros). Estos rangos, presentados en el modelo, posibilitarán la optimización de la liquidez, la capacidad de endeudamiento y la maximización del rendimiento. De comprobarse lo contrario, podría insertarse otra u otras variables (o Ratios), con características similares.

Los rangos valorativos (óptimos), de la propuesta, plantean la Razón de Liquidez, Razón de Endeudamiento y Razón de Rendimiento Financiero (ROE) (Tabla 7):

\footnotetext{
7. Los RATIOS son razones o relaciones entre dos magnitudes cuya efectividad depende de comparaciones múltiples. La relación se realiza entre dos números o índices del Balance o Estado de Resultados que mide diversos cambios sufridos en la organización durante el periodo.
}

8. Son los conceptos que parten de la hipótesis del Devengo y la continuidad, misma que explica los objetivos, características, hipótesis, elementos, reglas, valoración, entre otros, de los Estados Financieros. 


\section{Tabla 7.}

\section{Rangos valorativos considerados óptimos de la propuesta del modelo (MOADANIIF)}

\begin{tabular}{|c|c|c|c|c|c|c|}
\hline \multirow[t]{2}{*}{ Cooperativa } & \multicolumn{2}{|c|}{$\begin{array}{c}\text { Razón de Liquidez } \\
\text { (LIQ) } \\
\left(\mathbf{X}_{1}\right)\end{array}$} & \multicolumn{2}{|c|}{$\begin{array}{c}\text { Razón Endeudamiento } \\
\text { (END) } \\
\left(\mathbf{X}_{2}\right)\end{array}$} & \multicolumn{2}{|c|}{$\begin{array}{l}\text { Razón de Rendimiento } \\
\text { Financiero-ROE } \\
\left(\mathbf{X}_{3)}\right.\end{array}$} \\
\hline & $\begin{array}{c}\text { Alerta y/o } \\
\text { riesgo }\end{array}$ & Óptimo & $\begin{array}{c}\text { Alerta y/o } \\
\text { riesgo }\end{array}$ & Óptimo & $\begin{array}{c}\text { Alerta y/o } \\
\text { riesgo }\end{array}$ & Óptimo \\
\hline $\begin{array}{l}\text { Ahorro y Crédito y } \\
\text { SM integrales con } \\
\text { servicios financieros } \\
\text { Media: }\end{array}$ & $<1.1>2.5$ & $\begin{array}{c}\geq 1.1<2.5 \\
\left(\dot{X}_{1=1.8}\right)\end{array}$ & $<0.4>0.8$ & $\begin{array}{c}\geq 0.4<0.8 \\
\left(\dot{\mathbf{X}}_{2}=0.6\right)\end{array}$ & $<0.05$ & $\begin{array}{c}\geq 0.05 \\
\left(\dot{\mathbf{X}}_{3} \geq 0.05\right)\end{array}$ \\
\hline
\end{tabular}

Fuente: Elaboración propia, basada en Martínez, 2014a

Tal como se observa en la figura 1, primero, se busca con la propuesta la optimización re-expresando los valores del Ratio de Liquidez que se ubiquen entre 1.1 a 2.5 (Relación del Activo Circulante / Pasivo Circulante). En la tesis de Martínez (2014), se probó independencia entre las variables categóricas (Liquidez - norma plena) con las estudiadas (Liquidez - propuesta) mediante el chi-cuadrado y la t-student. Segundo, se busca la capacidad de pago con el Ratio de Endeudamiento ubicado entre 0.40 a 0.80 (Relación del Pasivo Total / Activo Total); es decir "medir el nivel global de endeudamiento 0 proporción de fondos de los acreedores" (Quito R., 2005, p. 9). Tanto así que en las cooperativas "los activos como los pasivo son la base para el fortalecimiento de sus clientes y asociados" (De Sárraga \& Spalding, 2002, p. 32). Y tercero, la maximización de las variables, igual o mayor, de 0.05 (Excedente neto de periodo / Capital Contable).

El Estudio de Caso, usado en esta investigación, supone incorporar otros argumentos que sustente la aplicabilidad de la propuesta. Sería analizada en estudios ulteriores.

\section{CONCLUSIÓN}

Es evidente que las Normas Internacionales de Contabilidad (NIC) y las Normas Internacionales de Información Financiera (NIIF), no se están usando de forma plena en las cooperativas; principalmente en el tema de instrumentos financieros. Con mayor fundamento en las cooperativas de I.F., sector objeto de este estudio.

Al referenciar la teoría, Al contrastar el escenario actual (real) versus la norma plena, se observan importantes diferencias en la situación financiera de las cooperativas de I.F. La aplicación radical de la norma plena, en términos de la totalidad del sector cooperativo del país, presenta desmejoramiento en el Patrimonio de $14.8 \%$ (de $24.8 \%$ a $10.0 \%$ ) y un endeudamiento general de $14.8 \%$ (de $75.2 \%$ a $90.0 \%$ ).

La relación del escenario actual, en el Estudio de Caso de las cooperativas de I.F. representativas del país, versus la norma plena, considerando el Capital mínimo irreducible de Aportaciones, presenta también desmejoramiento relevante del Patrimonio en $10.2 \%$ (de $17.1 \%$ a $6.9 \%$ ) y aumento significativo en los Pasivos Financieros de $10.2 \%$ (de $82.9 \%$ a $93.1 \%)$.

Estos efectos, lógicamente, modifican la estructura del Patrimonio (Aportaciones, Reservas, Excedentes).

Los Estados Financieros auditados, de los cinco (5) casos estudiados aquí, fueron considerados mediante el análisis vertical de los años 2015 (las tres sociedades solidarias de I.F. principales) y del 2012 y 2013 las dos restantes.

La Hipótesis planteada en la investigación: El posicionamiento patrimonial de las cooperativas de 
I.F. contrastan con el planteamiento teórico de las normas internacionales de información financiera NIC/NIIF, queda evidenciado el contraste con las diferentes comparaciones.

En definitiva, se requiere legislar sobre el tema para establecer líneas acordes con la estructura patrimonial coherente con estas sociedades solidarias y que no siga la incertidumbre. No hay que negar, existen diferencias abismales entre las cooperativas y las sociedades laborales, que si bien no tienen un fin lucrativo, si luchan por mantener la solidez empresarial. Significa que la gestión (manejo administrativo), en este tipo de sociedad, mide los indices desde otra perspectiva (enfoque social).

En la actualidad existe una Comisión interinstitucional (gobierno y sector) analizando las estrategias más viables para modificar la ley cooperativa del país. Es importante que además de las leyes nacionales, sea categorizada la norma contable internacional bajo otra figura técnica adaptada al sistema de sociedades cooperativas. Por mucho que el ente rector de las cooperativas en Panamá (IPAC00P) promueva la aplicación plena de las NIC/NIIF, no se pueden cumplir con algunas de ellas por lo ya planteado.

\section{REFERENCIAS BIBLIOGRÁFICAS}

Alianza Cooperativa Internacional para las Américas - ACI Américas (2016). Conferencia Latinoamericana sobre Gobernanza Cooperativa. Recuperado de: http://www. aciamericas.coop/

Amat, 0. \& Perramon, J. (2005). Normas Internacionales de Contabilidad-NIC/NIIF: Edición especial para Latinoamérica. Madrid. Gestión 2000.

Asociación Española de Contabilidad y Administración de Empresas - AECA (2012). Comisión de Contabilidad de Cooperativas. Recuperado de: http://www.aeca. es/comisiones/comisioncoop.htm

Berrio, G., G. L. (2015). Proceso de convergencia a NIIF en las cooperativas y sector cooperativo en Colombia. Recuperado de: http://www.aciamericas.coop/IMG/ pdf/microsoft_powerpoint_-_convergencia_a_niif_en_ las_cooperativas_y_sector_cooperativo_junio_03.pdf Comité de Interpretaciones de las Normas Internacionales de Información Financiera - CINIIF 2 (2010). Aportaciones de Socios de Entidades Cooperativas e Instrumentos similares. Recuperado de: https://www. mef.gob.pe/contenidos/conta_publ/con_nor_co/ vigentes/ciniif/2_CINIIF.pdf
Consejo de Normas Internacionales de Contabilidad IASB (2016). Recuperado de: http://www.ifrs.org/ Pages/default.aspx

Cubedo, M. (2007). El Régimen Económico de las Sociedades Cooperativas: Situación Actual y Apuntes para una Reforma. CIRIEC-España, Revista de Economía Pública, Social y Cooperativa, (58), 161-187. Recuperado de: http://redalyc.uaemex.mx/redalyc/ pdf/174/17405807.pdf

De Sárraga, A. \& Spalding, C. (2002). Disciplinas financieras para cooperativas de ahorro y crédito: soporte para la movilización de ahorros. Panamá. COLAC y Centro Cooperativo Sueco. Impresora Pacífico.

Gómez, A., P. \& Miranda, G, M. (2006). La caracterización financiera y contable del capital social a la luz de los principios cooperativos. REVESCO No.90. pp. 7-27. Recuperado de: http://eprints.ucm. es/10343/1/REVESCO_N_90.1_Pilar_GOMEZ\%2C_ MARTA_MIRANDA.pdf

Gómez, 0., De La Hoz A. \& De La Hoz, B. (2010). Armonización de las NIC/NIIF en las prácticas contables de entes emisores no financieros que cotizan en la Bolsa de Valores de Caracas, Venezuela. Contaduría y Administración, (233), 149-175. Recuperado de: http://redalyc.uaemex.mx/redalyc/ pdf/395/39515424009.pdf

Hernández S., R., Fernández C., C. \& Baptista L., P. (2007). Metodología de la Investigación. Cuarta edición. México. McGraw Hill.

IAS 32 (2012). Financial Instruments: Presentation. Technical Summary (32). Recuperado de: http://www. ifrs.org/Documents/IAS32.pdf

IAS 39 (2012). Financial Instruments: Recognition and Measurement. Technical Summary (39). Recuperado de: http://www.ifrs.org/IFRSs/Documents/English\%20 IAS\%20and\%20IFRS\%20PDFs\%202012/IAS\%2039.pdf IFRS 7. (2012). Financial Instruments: Disclosures. Technical Summary (7). Recuperado de: http://www. ifrs.org/IFRSs/Documents/IFRS7en.pdf

Ley 17 de 1997. Que establece un régimen especial para las cooperativas. Gaceta Oficial 23,279 de 5 de mayo de 1997. Panamá.

Martin, S., L., G. \& Iturrioz, J. (2007). La naturaleza del capital social como aspecto diferenciador entre las sociedades cooperativas y las sociedades laborales. CIRIEC-España, Revista de Economía Pública, Social y Cooperativa, (58), 59-82. Recuperado de: http://www.ciriec-revistaeconomia.es/ banco/5803_Martin_et_al.pdf 
Martínez, F. (2014a). Modelo de Adaptación de las Normas Internacionales de Información Financiera a la Estructura Patrimonial de las Cooperativas Panameñas. Tesis doctoral. Universidad de Panamá.

Martínez, F. (2014b). Modelo de Adaptación de las Normas Internacionales de Información Financiera a la Estructura Patrimonial de las Cooperativas Panameñas. Gestión Joven. Revista de la Agrupación Joven Iberoamericana de Contabilidad y Administración de Empresas (AJOICA), (12), 15-30. Recuperado de: http://www.elcriterio.com/revista/ contenidos_12/2\%20Art\%edculo\%20Francisco $\% 20$ Mart\%ednez.pdf

NIIF 7 (2015). Instrumentos Financieros: Información a Revelar (7). Recuperado de: https://www.mef.gob. pe/contenidos/conta_publ/con_nor_co/no_oficializ/ nor_internac/ES_GVT_IFRS07_2013.pdf

Polo G., F. (2006). La contabilidad de cooperativas en un proceso de armonización contable internacional: El caso de España. Madrid. Revesco - Revista de Estudios Corporativos, (89), 107-138. Recuperado de: http:// www.ucm.es/info/revesco/txt/REVESC0\%20N\%20 89.4\%20Fernando\%20POL0\%20GARRID0.htm
Polo, G, F. (2007). Impacto de las Normas Internacionales de Información Financiera en el Régimen Económico de las Sociedades Cooperativas. CIRIEC-España. Revista de Economía Pública, Social y Cooperativa, (058), 83-108 Recuperado de: http://redalyc.uaemex. $\mathrm{mx} / \mathrm{redalyc} / \mathrm{pdf} / 174 / 17405804 . \mathrm{pdf}$

Quito, R., C. (2005). Análisis de Estados Financieros. Revista Ratios, 40. Recuperado de: http://ucvvirtual. edu.pe/campus/HDVirtual/20010127/RATIOS\%20 FINANCIEROS/7000001541/RATIOS_FINANCIEROS.pdf Rendón-Álvarez, B., Rodríguez-Bolaños, J., \& RiascosGómez, P. A. (2013). Análisis del impacto en el capital institucional de las cooperativas de ahorro y crédito frente a la aplicación de las NIIF. Caso cooperativas del Valle del Cauca. Cuadernos de Contabilidad., 14 (36), 881-901. Recuperado de: http://www.scielo.org.co/pdf/cuco/v14nspe36/ v14nspe36a02.pdf 